\title{
miR-17-5p attenuates kidney ischemia-reperfusion injury by inhibiting the PTEN and BIM pathways
}

\author{
Ming $\mathrm{Ma}^{1,2 \#}$, Lei Fu ${ }^{3 \#}$, Zihao Jia ${ }^{1,2}$, Qiang Zhong ${ }^{1,2}$, Zhongli Huang ${ }^{1,2}$, Xianding Wang ${ }^{1,2}$, Yu Fan ${ }^{1,2}$, \\ Tao Lin ${ }^{1,2}$, Turun Song ${ }^{1,2}$ \\ ${ }^{1}$ Urology Department, West China Hospital, Sichuan University, Chengdu, China; ${ }^{2}$ Organ Transplantation Center, West China Hospital, Sichuan \\ University, Chengdu, China; ${ }^{3}$ Urology Department, The Third People's Hospital of Chengdu, Chengdu, China \\ Contributions: (I) Conception and design: T Song, T Lin; (II) Administrative support: T Song, T Lin; (III) Provision of study materials or patients: M \\ Ma, L Fu, Z Jia, Q Zhong; (IV) Collection and assembly of data: M Ma, L Fu, Z Huang, Y Fan; (V) Data analysis and interpretation: M Ma, L Fu, Q \\ Zhong, X Wang; (VI) Manuscript writing: All authors; (VII) Final approval of manuscript: All authors. \\ "These authors contributed equally to this work and should be considered as co-first authors. \\ Correspondence to: Turun Song; Tao Lin. West China Hospital, 37\# Guoxue Alley, Chengdu 610041, China. \\ Email: 21062068@scu.edu.cn; kidney5@163.com.
}

Background: Kidney ischemia-reperfusion (I/R) injury is an independent risk factor for delayed graft function after kidney transplantation with long-term graft survival deterioration. Previously, we found that the upregulated expression of miR-17-5p exerts a protective effect in kidney I/R injury, but the mechanism has not been clearly studied.

Methods: A kidney I/R injury model was induced in adult C57BL/6 male mice (20-22 g) by clamping both kidney pedicles for $30 \mathrm{~min}$. The miR-17-5p agomir complex was injected into mice 24 h before surgery via the tail vein at a total injection volume of $10 \mu \mathrm{L} / \mathrm{g}$ body weight. The mice were euthanized on post-I/R injury day 2, and kidney function, apoptosis, autophagy, and related molecules were then detected. Human kidney-2 (HK-2) cells, which underwent hypoxia/reoxygenation, were treated with the miR-17-5p agomir, miR-17-5p antagomir, and small interfering ribonucleic acids (siRNAs). Cell viability, apoptosis, autophagy, and molecules were also examined.

Results: Autophagy, miR-17-5p expression, and kidney function damage were significantly more increased in the I/R group than in the sham group. In the cultured HK-2 cells underwent hypoxia/reoxygenation, the miR-17-5p agomir directly inhibited the expression of phosphatase and tensin homolog deleted on chromosome 10 (PTEN) and Bcl-2 like protein 11 (BIM), and attenuated apoptosis and autophagy. Further, miR-17-5p inhibited autophagy by activating the protein kinase B (Akt)/Beclin1 pathway, which was suppressed by siRNAs. Additionally, the administration of miR-17-5p agomir greatly improved kidney function in the I/R mice group by inhibiting autophagy and apoptosis.

Conclusions: These findings suggest a new possible therapeutic strategy for the prevention and treatment of kidney I/R injury. The upregulation of miR-17-5p expression appears to inhibit apoptosis and autophagy by suppressing PTEN and BIM expression, which in turn upregulates downstream Akt/Beclin1 expression.

Keywords: Autophagy; Bcl-2 like protein 11 (BIM); microRNA; phosphatase and tensin homolog deleted on chromosome 10 (PTEN); kidney ischemia-reperfusion injury (I/R injury)

Submitted Aug 13, 2021. Accepted for publication Sep 27, 2021.

doi: $10.21037 /$ atm-21-4678

View this article at: https://dx.doi.org/10.21037/atm-21-4678 


\section{Introduction}

Kidney ischemia-reperfusion (I/R) injury is characterized by a transient cessation and restoration of blood flow, and reoxygenation is associated with a significantly increased incidence of morbidity and mortality in major surgical procedures $(1,2)$. Even after recovery from the initial injury, kidney I/R injury may still result in chronic kidney disease or even kidney failure (3). Further, it is an independent risk factor for delayed graft function after kidney transplantation with long-term graft survival deterioration (4). Kidney I/R injury is an intriguing pathophysiological process that involves inflammation, immunological responses, oxidative stress, tubular injury, and endothelial dysfunction (5). However, the mechanism is still not well elucidated, and limited therapies are available for the effective treatment of I/R-induced kidney injury.

Autophagy plays a vital role in kidney I/R injury (6-8). The phosphatase and tensin homolog deleted on chromosome 10 (PTEN), phosphatidylinositol-3 kinase (PI-3K), protein kinase B (Akt), Bcl-2 like protein 11 (BIM), Beclin1, and adenosine monophosphate-activated protein kinase (AMPK)/mammalian target of rapamycin (mTOR) pathways may be key signaling molecules (6). However, research on the effects of autophagy on kidney I/R injury has produced conflicting results $(9,10)$. It is hypothesized that autophagy plays a dual role in the kidney I/R process, having both protective and detrimental properties, depending on the duration of the ischemic period and the phase of I/R injury (11).

Micro ribonucleic acids (miRNAs) have recently been recognized as small non-coding RNAs, approximately 21-25 nucleotides in length. They post-transcriptionally regulate gene expression. The specific ablation of Dicer (a key enzyme for miRNA production) leads to kidney diseases and dysfunction, which indicates the critical roles of the miRNAs involved in kidney development and physiological maintenance $(12,13)$. Fifteen differentially expressed miRNAs were identified in the kidneys of a rat kidney I/ R injury model, and miR-10a, miR-192, and miR-194 were found to be significantly increased in the plasma of the rats (14). Recent studies have also investigated other miRNAs in kidney I/R injury, and found that miR-20, miR-126, and miR-489 may protect kidneys from I/R injury (15-17).

MiR-17-92 is a highly conserved cluster that encodes 6 miRNAs (miR-17, miR-18a, miR-19a, miR-19b-1, miR20a, and miR-92a). Postnatally, the early development of focal glomerulosclerosis and podocyte foot process effacement is detected at 3 months, which indicates that miR-17-92 is necessary for nephrogenesis and kidney function (18). MiR-17-92 might mediate the progression of cysts in polycystic disease, and the miR-17-mediatedinhibition of mitochondrial metabolism might be the key regulating pathway $(19,20)$. Previously, we showed that the expression of the miR-17-92 cluster (especially the miR17-5p) increases after kidney I/R injury, and the deletion of miR-17-92 in proximal tubule cells makes the kidneys highly susceptible to I/R injury (21). However, the exact mechanism of miR-17-92 in kidney I/R injury remains unknown. We present the following article in accordance with the ARRIVE reporting checklist (available at https:// dx.doi.org/10.21037/atm-21-4678).

\section{Methods}

\section{Murine model of kidney I/R injury}

Twenty-four 6-8-week-old (20-22 g) C57BL/6 male mice (Chengdu Dossy Experimental Animals, Co., Ltd., Chengdu, China) were included in this study. Using the random number method, the mice were randomly divided into a sham group $(\mathrm{n}=8)$, I/R group $(\mathrm{n}=8)$, and $\mathrm{I} / \mathrm{R}+$ agomir group $(n=8)$. The mice were fed adaptively for 7 days, and then anaesthetized with intraperitoneal pentobarbital (30 $\mathrm{mg} / \mathrm{kg}$ ). Kidney I/R injury modeling was induced. In brief, bilateral dorsal incisions were made to expose kidney pedicles and both pedicles were clamped for $30 \mathrm{~min}$. The clamps were then released for reperfusion for 48 hours. Mice in the sham group were operated on to expose the kidney pedicles without clamping. The mice were raised under standard guidelines (4 mice per cage), and all mice were monitored throughout the experiment. The mice were free of viral, bacterial, and parasitic pathogens, and all mice survived before they were euthanized at the end of the experiment. The Animal Ethics Committee of West China Hospital, Sichuan University, Chengdu, China (No. 2019141A) approved this study. All animal studies were conducted in accordance with the Guide for the Care and Use of Laboratory Animals of the National Institutes of Health. A protocol was prepared before the study without registration.

\section{In vivo miR-17-5p agomir delivery}

The miR-17-5p agomir and agomir-negtive control (NC) were synthesized by GenePharma Co. Ltd. (Shanghai, China) 
and delivered as per the protocol of the Invivofectamine 2.0 kit (Invitrogen, Carlsbad, CA, USA). In brief, $200 \mu \mathrm{L}$ of Invivofectamine-agomir complex was prepared by mixing the miRNA agomir $(20 \mathrm{nmol})$ and Invivofectamine at the ratio of 1:1. For the in-vivo test, the Invivofectamine-agomir complex $(n=4)$ or agomir-NC complex $(n=4)$ were injected into mice $24 \mathrm{~h}$ before I/R surgery via the tail vein at $20-40 \mu \mathrm{L} / \mathrm{s}$ at a total injection volume of $10 \mu \mathrm{L} / \mathrm{g}$ body weight.

\section{Histological evaluation and TUNEL assay}

The mice were euthanized after I/R for 48 hours, and their kidneys were perfused with phosphate-buffered saline (PBS) followed by $4 \%$ paraformaldehyde. The kidneys were fixed in $10 \%$ formalin and then embedded in paraffin. The sections were cut and stained with hematoxylin and eosin. Histological changes, including tubular necrosis, tubular dilation, cast formations, and the loss of brush border, were examined in a blinded manner. 10 areas in randomly selected kidney tubules from the outer medulla of the kidney were examined at $\times 200$ magnification. Based on the percentage of the injury area, pathological scores were assigned on a scale of 0 to 5 (on which 0 points represented normal, 1 point represented an injury area $<10 \%, 2$ points represented an injury area between $10-25 \%, 3$ points represented an injury area between $25-50 \%, 4$ points represented an injury area between $50-75 \%$, and 5 points represented an injury area $>75 \%$ ) (22). Micrographs of the representative fields were recorded. Apoptosis in kidney tissues was identified using the terminal deoxynucleotidyl transferase-mediated dUTP nick-end labeling (TUNEL) assay with a Cell Death Detection kit (Roche Applied Science, IN, USA). Five fields per section and 3 sections per kidney were examined in each experimental group ( $\mathrm{n}=4$ mice in each group).

\section{Kidney function analysis}

Kidney function $(n=4)$ was monitored by measuring blood urea nitrogen (BUN) and serum creatinine (Scr). In brief, blood samples were collected and centrifuged. BUN and Scr were measured using commercial kits from Nanjing Jiancheng Bioengineering Institute (Jiangsu, China).

\section{Cell culture and bypoxia modeling}

The human kidney-2 (HK-2) cell line was purchased from Chinese Type Culture Collection (CTCC). The HK-2 cells were cultured in a medium containing $10 \%$ fetal bovine serum (FBS; Hyclone, Logan, UT, USA) and a 5\% carbon dioxide $\left(\mathrm{CO}_{2}\right)$ chamber (Thermo Fisher Scientific Inc. Waltham, MA, USA), and moved to hypoxia treatment after growing to cover $80 \%$ of the plate. The cells were plated in 96-cell plates with $2 \times 10^{3}$ cells in complete medium overnight, changed to Dulbecco's Modified Eagle Medium (DMEM; Hyclone, Logan, UT, USA) without FBS, and incubated in a hypoxia chamber with $95 \%$ nitrogen $\left(\mathrm{N}_{2}\right)$ and $5 \% \mathrm{CO}_{2}$ for 6,12 , and $24 \mathrm{~h}$. The cells were then cultured in a medium containing $10 \% \mathrm{FBS}$ and a $5 \% \mathrm{CO}_{2}$ chamber for 6,12 , and $24 \mathrm{~h}$. After the initial experiments, we determined that hypoxia for $24 \mathrm{~h}$ and reoxygenation for $24 \mathrm{~h}$ represented reasonable times to detect apoptosis and cell viability.

\section{Flow cytometry}

Flow cytometry was used to detect the cell apoptosis rate. In brief, cells were digested with trypsin without ethylenediaminetetraacetic acid, centrifuged at $2,000 \mathrm{rpm} / \mathrm{min}$ for $5 \mathrm{~min}$. The supernatant was discarded and the cells were collected. After washing the cells twice with pre-chilled PBS, $300 \mu \mathrm{L}$ of Binding Buffer was added to the cell precipitate to suspend the cells. Add $5 \mu \mathrm{L}$ of Annexin $\mathrm{V}$-FITC to the cell suspension and then incubate for $15 \mathrm{~min}$ at room temperature and protected from light. After this, $10 \mu \mathrm{L}$ of PI stain was added to the cell suspension and incubate for $10 \mathrm{~min}$ at room temperature under light-proof conditions. Finally, apoptosis was detected by using flow cytometry and analyzed by CELL Quest software.

\section{Cell counting kit-8 (CCK-8) assay}

Cell viability was assessed with the CCK-8 assay in accordance with the manufacturer's instructions (Beyotime Biotechnology Co., Ltd., Haimen, China). The HK-2 cells were plated in 96-well plates at a density of 2,000 cells/ plate and cultured for $24 \mathrm{~h}$. After hypoxia/reoxygenation treatment, the culture medium was replaced with 110 $\mu \mathrm{L}$ of CCK-8 solution (containing $100 \mu \mathrm{L}$ of serum-free DMEM and $10 \mu \mathrm{L}$ of CCK-8 reagent). After incubating the cells for $4 \mathrm{~h}$, we measured the absorbance in each well at a wavelength of $450 \mathrm{~nm}$ with a microplate spectrophotometer (MK3, Thermo).

\section{Measurement of autophagosomes}

The HK-2 cells grown on the coverslips were transfected 
with mRFP-GFP-LC3 adenovirus (Hanheng Bio, Shanghai, China), administered additional treatments for appropriate periods, and subjected to hypoxia/reoxygenation treatment. The cells were then fixed with $10 \%$ formalin. RFPGFP-LC3 fluorescence was observed under a confocal fluorescence microscope (Leica, Heidelberg, Germany).

\section{Real-time polymerase chain reaction (PCR)}

In accordance with the manufacturer's instructions, total RNA from the cells and kidney tissues $(n=4)$ was extracted using Trizol (Invitrogen). Subsequently, the total RNA (2 $\mathrm{mg}$ ) was converted to complementary deoxyribonucleic acid (cDNA) using the miRNA Reverse Transcription Kit (Thermo \#K1622, MA, USA). The real-time PCR was performed using the SYBR Green PCR kit (Thermo F-415XL, MA, USA), which included sequence-specific primers for the cDNA synthesis and probes for the realtime PCR. Fold changes were quantified using $2-\Delta \Delta \mathrm{Ct}$ values. The primer sets are set out in Tables S1-S4.

\section{Luciferase reporter assay}

TargetScan (www.targetscan.org) was used to forecast the potential miR-17-5p biological target sites. To construct a luciferase reporter vector, we synthesized the PTEN3'UTR and BIM-3'UTR, which contained the binding region of miR-17-5p, and then inserted these fragments into a pYr-Mir Target (XhoI/NotI) vector to form the pYr-MirTarget-PTEN-3 'UTR ${ }^{\mathrm{WT}}$ and pYr-MirTargetBIM-3'UTR ${ }^{\mathrm{WT}}$ luciferase vector (Hangzhou Luepu bio, Hangzhou, China). The 3 prime untranslated region (3'UTR) mutant (MUT) luciferase vectors of PTEN and BIM were constructed into pYr-MirTarget-PTEN$3^{\prime} \mathrm{UTR}^{\mathrm{MUT}}$ and pYr-MirTarget-BIM-3'UTR ${ }^{\mathrm{MUT}}$. For the reporter assay, we co-transfected the HK-2 cells that were seeded in 24-well plates with either luciferase reporter construct, or the miR-17-5p agomir (Invitrogen). $48 \mathrm{~h}$ after transfection, the cells were lysed, and luciferase activity levels were measured using a dual-luciferase reporter assay system (Promega, Madison, USA). Firefly luciferase activity levels were normalized to Renilla luciferase activity levels. The primer sets are set out in Tables S1-S4.

\section{miR-17-5p-agomir or antagomir transfection and $R N A$ interference}

The miR-17-5p-agomir (50 nM) or the miR-17-5p antagomir (100 $\mathrm{nM})$ was transfected into the HK-2 cells for $48 \mathrm{~h}$ to upregulate or downregulate miR-17-5p expression using Lipofectamine 3000 (Invitrogen) in accordance with the manufacturer's instructions. The expression of PTEN, BIM, and Akt were also reduced by transfection with corresponding small interfering ribonucleic acids (siRNAs) (50 nM, Jima Pharm, Shanghai, China). All interventions were paired with the corresponding control group.

\section{Molecular examination}

A immunoblot analysis was conducted following a standard protocol. In brief, the whole cell lysate was collected by using 2\% sodium dodecyl sulphate (SDS) buffer (Biosharp, Hefei, Anhui, China). The exact amount $(30 \mu \mathrm{g})$ of protein samples was resolved by SDS-polyacrylamide gel electrophoresis (PAGE) gel (Sigma-Aldrich, St. Louis, MO, USA), and then transferred to the polyvinylidene fluoride (PVDF) membranes (Merck Millipore, Billerica, MA, USA). The blots were blocked in 5\% non-fat milk for $1 \mathrm{~h}$ before incubation in primary and secondary antibodies. The antibodies were purchased from the following sources: LC3 (1:500, Ab51520, Affinity Biosciences, Cincinnati, OH, USA), P62 (1:500, Ab56416, Affinity), PTEN (1:1,000, AF6351, Affinity), BIM (1:1,500, AF0121, Affinity), BMPR2 (1:1,000, BF0342, Affinity), Beclin1 (1:1,000, AF7885, Affinity), p-Beclin1 (234) (1:250, ab183335, Abcam, Cambridge, UK), p-Beclin1(295) (1:250, ab183313, Abcam), Akt (1:1,000, AF6261, Affinity), GAPDH (1:1,000, Ab8245, Abcam), and p-Akt (1:1,000, AF0016, Affinity). To ensure that Western blot was in a linear range, we performed Western blot with a 2 -fold serial dilution of samples (from 1.25-80 $\mu$ g), and found that $30 \mu \mathrm{g}$ was within the linear range. The images were collected by ChemiScope 5300 Pro (Clinx Science Instruments Co., Ltd., Shanghai, China), and then quantified by Image J software (National Institute of Health, Bethesda, MD, USA).

\section{Transmission electron microscopy}

Kidney samples $(\mathrm{n}=4)$ of approximately $1 \mathrm{~mm}^{3}$ were clipped by ophthalmic scissors and immersed in $2.5 \%$ glutaraldehyde for $2 \mathrm{~h}$. The tissues were then washed 3 times with $0.1 \mathrm{M}$ phosphate buffer and fixed with $1 \%$ osmium tetroxide in $0.1 \mathrm{M}$ phosphate buffer for another $2 \mathrm{~h}$. The samples were dehydrated with increasing concentrations of ethanol and $100 \%$ acetone, and then embedded in Embed 812 (Electron microscopy science). 
Semi-thin sections were cut at $1 \mu \mathrm{m}$ and stained with $1 \%$ toluidine blue to evaluate preservation quality. Ultrathin sections $(50-70 \mathrm{~nm})$ were cut, and mounted on copper grids. The autophagosome ultrastructure was observed under a transmission electron microscope (JEM-1200EX, JEOL Ltd., Tokyo, Japan).

\section{Statistical analysis}

The continuity variables are presented as mean \pm standard deviation (SD). Differences between the 2 groups were evaluated using the Student's $t$-test. For the multiple groups, the one-way NOVA analysis of variance followed by a Tukey test was used to compare the differences of all the column pairs. A $\mathrm{P}$ value $<0.05$ was considered statistically significant.

\section{Results}

\section{Autophagy was upregulated during kidney $I / R$ in vivo and bypoxia in vitro}

Kidney I/R injury was induced in mice by $30 \mathrm{~min}$ of bilateral kidney ischemia followed by reperfusion for $48 \mathrm{~h}$. The histological examination (see Figure 1A) showed severe tissue injury accompanied by increased autophagosomes in the I/ $\mathrm{R}$ group. The increase of BUN and Scr suggested that the $\mathrm{I} / \mathrm{R}$ model was completed, and an increased expression of miR-17-5p was observed (see Figure 1B). The expression of the LC3 II/I ratio was significantly upregulated after $48 \mathrm{~h}$ of reperfusion, while the expression of P62 was significantly decreased (see Figure 1C). In the HK-2 cells cultured in hypoxia, prolonging the reoxygenation time decreased the expression of miR-17-5p (see Figure 1D) and P62 (see Figure 1E), but increased the LC3 II/I ratio (see Figure 1E).

\section{miR-17-5p reduced apoptosis and autophagy in $H K-2$ cells}

To test the effects of miR-17-5p on kidney tubular cell apoptosis, the HK-2 cells were treated with the miR-17$5 \mathrm{p}$ agomir or antagomir. The transfection efficiency was high (see Figure $2 A$ ). Hypoxia intubation significantly impaired the viability and proliferation of the HK-2 cells, but the miR-17-5p agomir ameliorated this impairment. Conversely, the miR-17-5p antagomir aggravated hypoxiainduced impairment (see Figure 2B). Notably, transfection with the miR-17-5p antagomir almost increased the percentage of apoptotic cells by $20 \%$ under hypoxia, while the transfection of the miR-17-5p agomir significantly reversed this effect (see Figure 2C). As Figure 2D shows, the autophagy levels (the expression of P62 and LC3 II/I ratio) were significantly reduced after treatment with the miR-17$5 \mathrm{p}$ agomir; these results were confirmed by autophagy flux detection (see Figure 2E). When chloroquine (CQ, Sigma, C6628), an autophagy inhibitor, was used to treat the HK-2 cells, the autophagy levels of the HK-2 cells after hypoxia were significantly inhibited, and this effect was replicated with the miR-17-5p agomir. Additionally, we noted a more significant reduction in autophagy levels when the HK-2 cells were simultaneously treated with CQ and miR-17-5p agomir (see Figure 2F). The flow cytometry results indicated that CQ reduced $50 \%$ of the apoptosis of the HK-2 cells after hypoxia. A similar effect was observed in miR-17$5 \mathrm{p}$ agomir treatment. When the HK-2 cells were treated with CQ and the miR-17-5p agomir, a more significant reduction in apoptosis was noted in those cells than in those treated CQ or miR-17-5p only (see Figure 2G). These results suggest that miR-17-5p protects HK-2 cells from hypoxia-induced injury by inhibiting autophagy and apoptosis.

\section{miR-17-5p targeted PTEN and BIM in HK-2 cells}

Studies have suggested a close link among bone morphogenetic protein type II receptor (BMPR2), BIM, PTEN, and autophagy (23-25). Additionally, miR-17 inhibits the expression of PTEN, BIM, and BMPR2 (26-28). The bioinformatic analysis indicated that PTEN, BIM, and BMPR2 are targets of miR-17-5p, whose binding site is in the 3'UTR of PTEN, BIM, and BMPR2 (see Figure 3A). The miR-17-5p agomir downregulated the overexpression of PTEN and BIM induced by hypoxia, but did not change the expression of BMPR2 (see Figure 3B). The miRNA target luciferase reporter assay was applied to determine whether miR-17-5p directly targeted the 3'UTR of PTEN and BIM. The HK-2 cells were co-transfected with the luciferase reporter gene along with the miR-17-5p agomir. The miR-17-5p agomir significantly reduced luciferase expression in the luciferase-PTEN-3'UTR and luciferaseBIM-3'UTR transfected cells but not in the luciferasePTEN-3' TTR $^{\mathrm{MUT}}$ and luciferase-BIM-3 'UTR ${ }^{\mathrm{MUT}}$ transfected cells (see Figure 3C). Thus, miR-17-5p directly targeted PTEN-3'-UTR and BIM-3'UTR. Further, to determine the role of PTEN and BIM in the HK-2 cells that underwent hypoxia, we designed overexpression plasmids pcDNA3.1-PTEN and pcDNA3.1-BIM. A Western blot assay showed that pcDNA3.1-PTEN and 

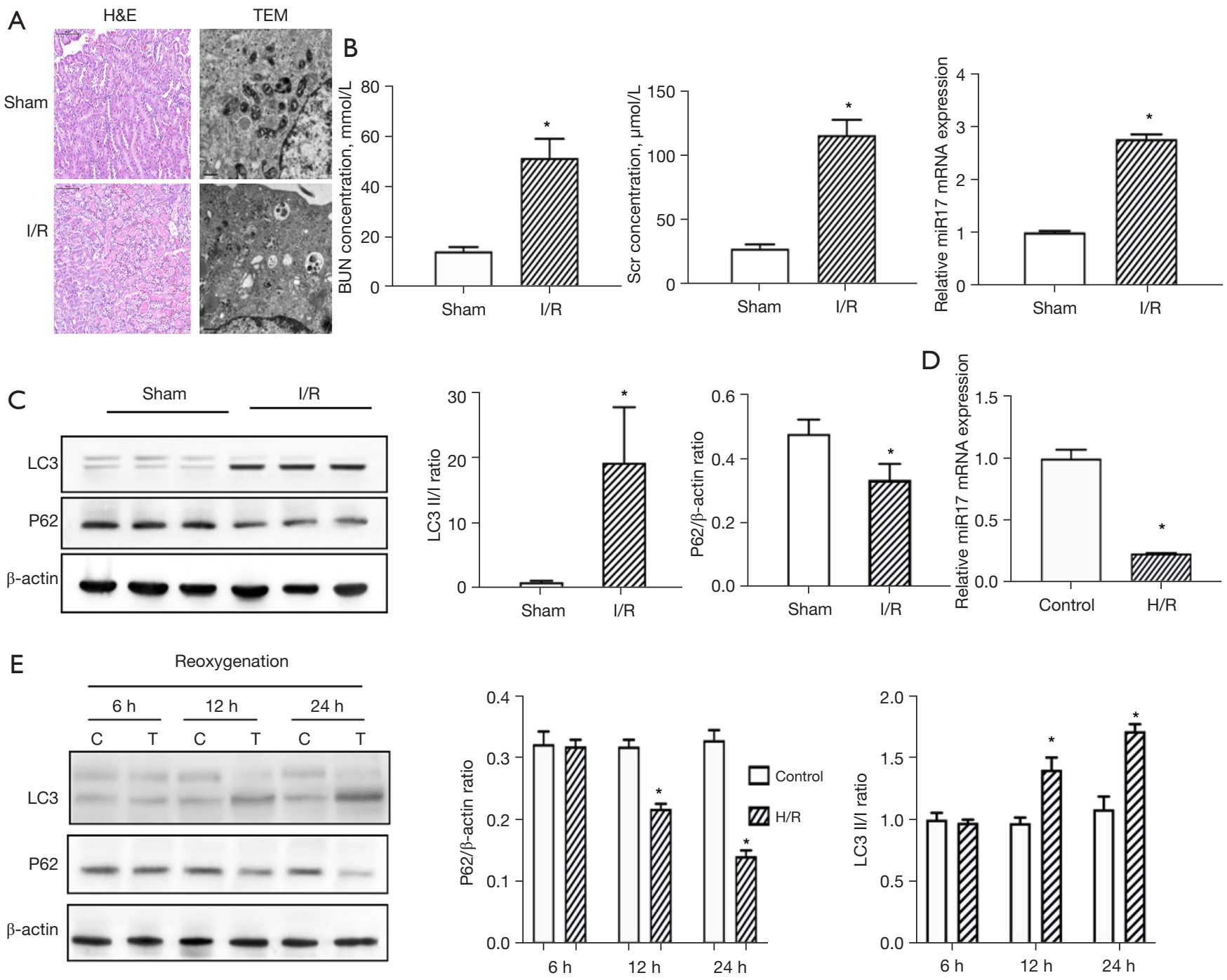

Figure $1 \mathrm{I} / \mathrm{R}$ injury upregulated the miR-17-5p expression and enhanced autophagy. Representative images (A) of kidney histology with H\&E staining and TEM ( $n=4)$. The concentration of BUN and Scr in mice, and the expression of miR-17-5p in mice kidneys ( $\mathrm{n}=4)(\mathrm{B})$. The expression of P62 decreased in the I/R group, LC3II increased, and LC3I decreased, indicating an increased autophagy level ( $\mathrm{n}=4$ ) (C). The expression of miR-17-5p in HK-2 cells (D). Representative band and statistical analysis of P62 and LC3 II/I expression in the HK-2 cells experiencing different times of H/R (E). *, P<0.05. BUN, blood urea nitrogen; H\&E, hematoxylin-eosin; H/R, hypoxia/ reoxygenation; HK-2, human kidney-2; LC3, microtubule-associated protein light chain 3; Scr, serum creatinine; TEM, transmission electron microscope.

pcDNA3.1-BIM significantly increased the expression of PTEN and BIM (see Figure 3D). Compared with the empty vector, pcDNA3.1-PTEN and pcDNA3.1-BIM significantly increased the apoptosis (see Figure $3 E$ ) and autophagy levels (see Figure 3F) of the HK-2 cells after hypoxia, and the miR-17-5p agomir partially reduced these upregulations. Thus, it appears that miR-17-5p protects HK-2 cells by repressing PTEN and BIM.

\section{miR-17-5p regulated the Akt/Beclin1 signaling pathway in $\mathrm{HK}-2$ cells}

Akt is the critical downstream signaling factor of PTEN and BIM, and the activation of Akt ameliorates I/R-induced kidney injury (29-31). After hypoxia or miR-17-5p agomir treatment, the expression of Akt produced no significant change compared to the control group. However, hypoxia 

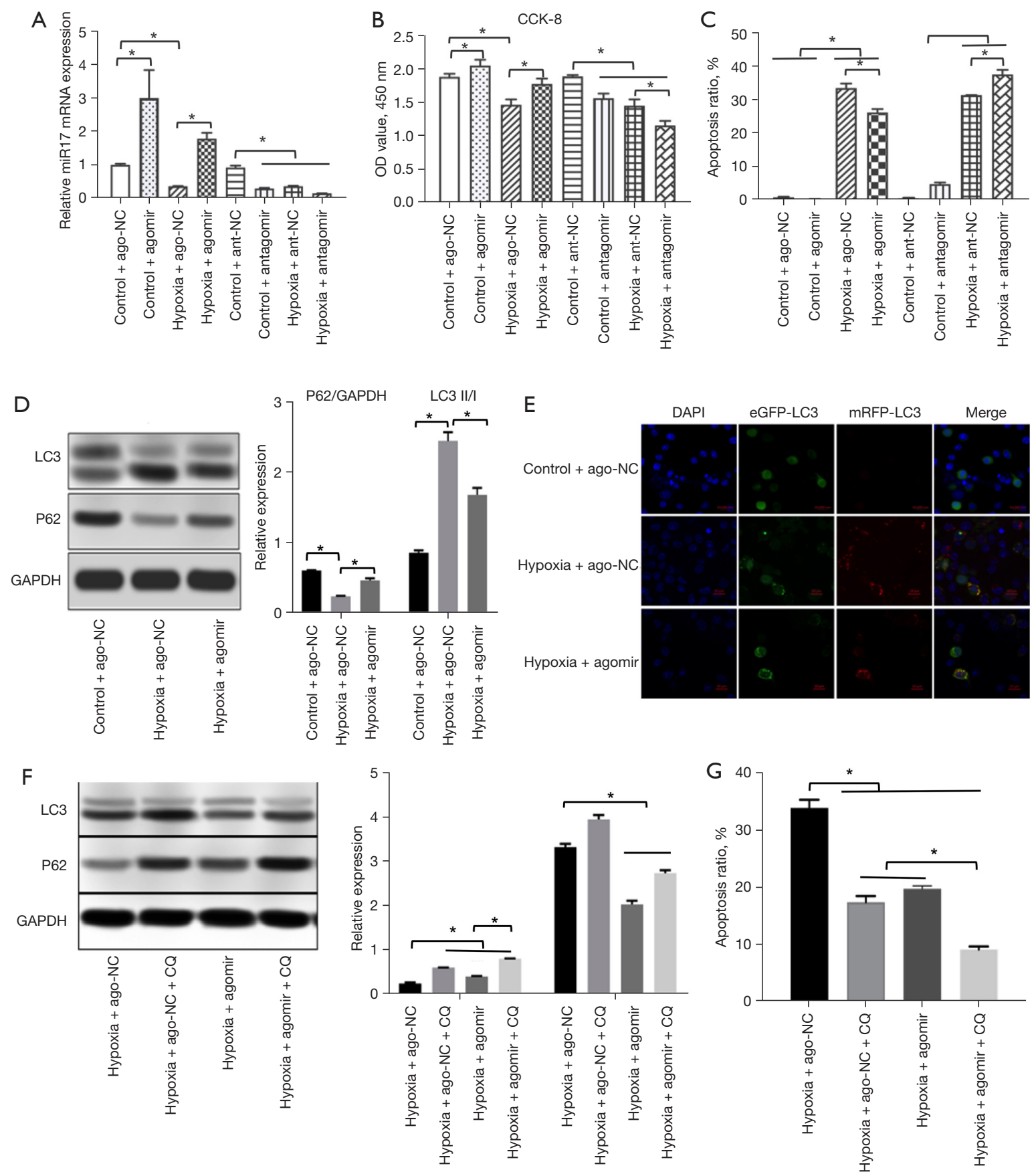

Figure 2 HK-2 cells were transfected with a miR-17-5p agomir (50 nM), and miR-17-5p antagomir (100 nM). HK-2 cells were incubated under hypoxia for 24 hours, and reoxygenated for 24 hours. The expression of miR-17-5p in HK-2 cells (A). Cell viability was detected by CCK-8 (B). Apoptosis was detected by flow cytometry (C). Representative band and statistical analysis (D) of LC3B II/I and P62 expression in HK-2 cells using Western blotting. Confocal microscopy and the mRFP-eGFp-LC3 system were used to analyze the autophagy flux. The punctuated red spot is autolysosome, and the punctuated yellow spot is autophagosome (E). Representative band and statistical analysis (F) of LC3B II/I and P62 expression in HK-2 cells. Apoptosis was detected by flow cytometry (G). * $\mathrm{P}<0.05$. Ago-NC, agomir-negative control; ant-NC, antagomir-negative control; CCK-8, cell counting kit-8; CQ, chloroquine; DAPI, 4',6-diamidino-2-phenylindole; GAPDH, glyceraldehyde phosphate dehydrogenase; GFP, green fluorescent protein; HK-2, human kidney-2; LC3, microtubule-associated protein light chain 3; RFP, red fluorescent protein. 
A

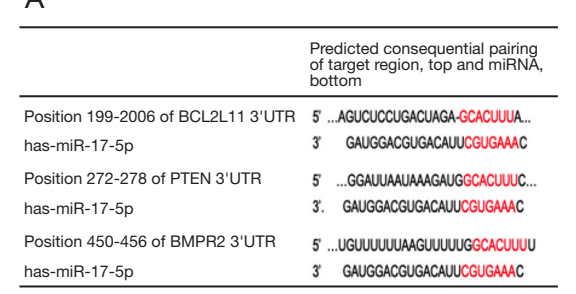

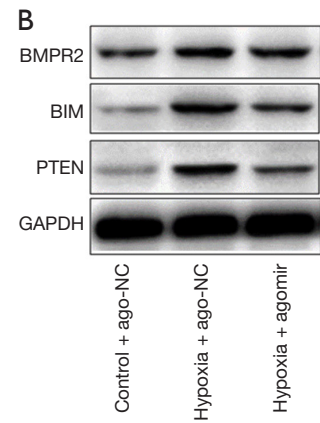

D

C

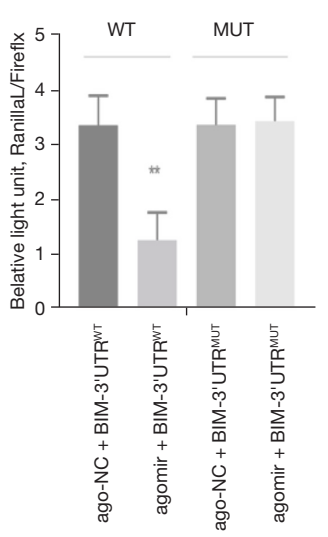

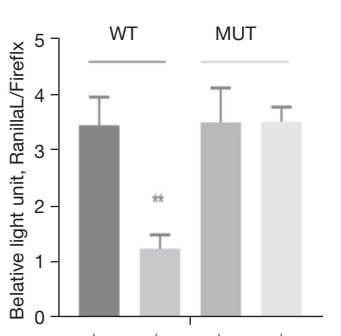

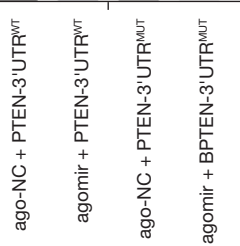

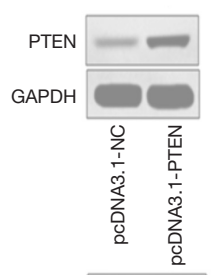

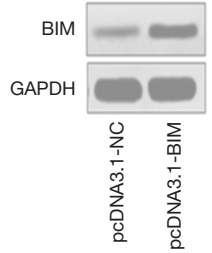
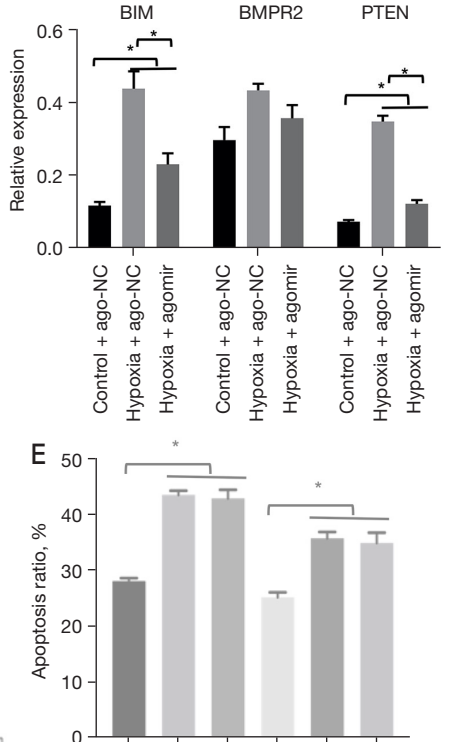

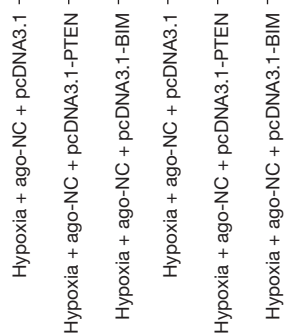

F
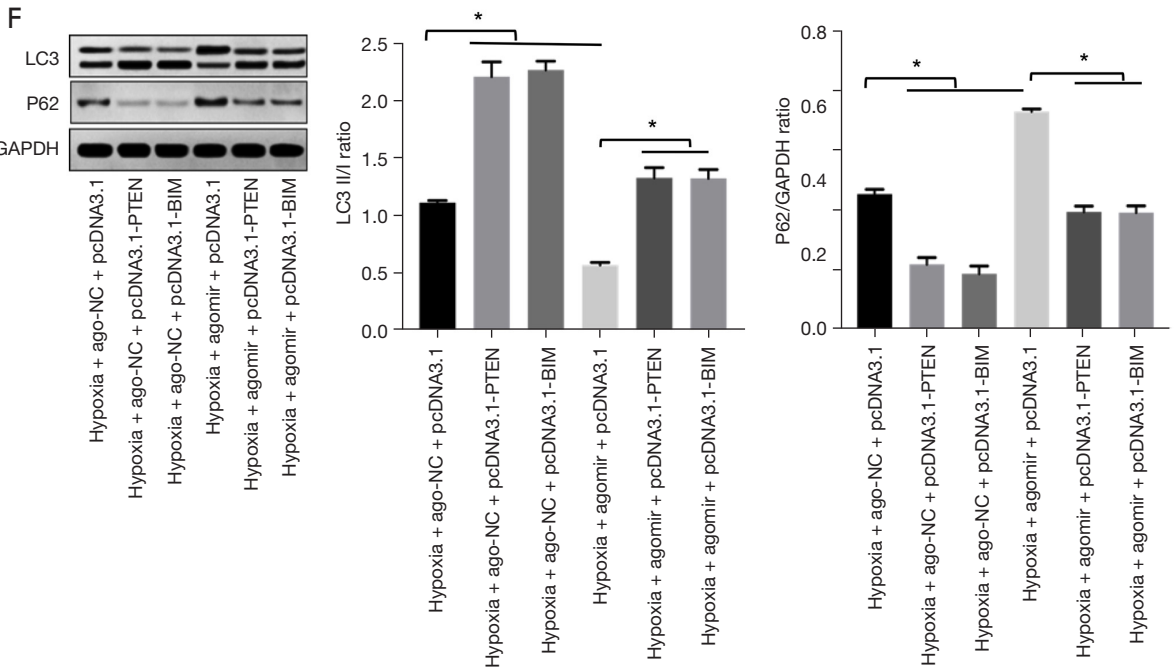

Figure 3 HK-2 cells were co-transfected with the Luciferase-PTEN-3'-UTR, Luciferase-BIM-3'UTR or Luciferase-PTEN-3'UTR ${ }^{\text {MUT }}$, Luciferase-BIM-3'UTRMUT along with the miR-17-5p agomir. The bioinformatic analysis (A) predicted potential targets of miR-17-5p. Representative band and statistical analysis (B) of BIM and PTEN expression in HK-2 cells. The luciferase expression (C) among transfected HK-2 cells were detected by quantitative real-time PCR. HK-2 cells were transfected with plasmid pcDNA3.1-PTEN and pcDNA3.1BIM. Western blot (D) confirmed the pcDNA3.1-PTEN and pcDNA3.1-BIM significantly upregulated the expression of PTEN and BIM. Flow cytometry detected the apoptosis percentage (E) of HK-2 cells. Representative band and statistical analysis (F) of LC3B II/I and P62 expression in HK-2 cells. *, $\mathrm{P}<0.05$. **, $\mathrm{P}<0.01$. ago-NC, agomir-negative control; BIM, Bcl-2 like protein 11; BMPR2, bone morphogenetic protein type II receptor; GAPDH, glyceraldehyde phosphate dehydrogenase; HK-2, human kidney-2; LC3, microtubule-associated protein light chain 3; MUT, mutant; PTEN, phosphatase and tensin homolog deleted on chromosome 10; siRNA, small interfering RNA. 
treatment dramatically reduced the expression of p-Akt, while the miR-17-5p agomir partially reversed this effect (see Figure 4A). Further, hypoxia increased the expression of Beclin1, but the expression of both phosphorylated forms p-Beclin1 (295) and p-Beclin1 (234) decreased. Additionally, the miR-17-5p agomir inhibited the expression of Beclin1 but increased the level of p-Beclin1 (295) and p-Beclin1 (234) (see Figure 4A). The HK-2 cells were subjected to hypoxia, and then pcDNA3.1-PTEN and pcDNA3.1-BIM significantly reduced the expression of p-Akt, p-Beclin1 (295), and p-Beclin1 (234) without changing the expression of Akt and Beclin1, while PTENsiRNA and BIM-siRNA significantly increased the expression of p-Akt, p-Beclin1 (295), and p-Beclin1 (234) (see Figure 4B). The expression of p-Akt decreased when the HK-2 cells were treated with Akt-siRNA, while the miR-17-5p agomir increased the expression of p-Akt. When the HK-2 cells were treated with the miR-17-5p agomir + Akt-siRNA, the expression enhancement of p-Akt was remarkably reversed (see Figure 4C). Further study indicated that Akt-siRNA significantly increased the autophagy level of the HK-2 cells that underwent hypoxia, and significantly reversed the autophagy inhibition effect of the miR-17$5 \mathrm{p}$ agomir (see Figure 4D). The flow cytometry results also demonstrated that Akt-siRNA significantly increased the apoptosis of the HK-2 cells and reversed the protective effect of miR-17-5p agomir (see Figure 4E). These results suggest that miR-17-5p might inhibit autophagy and apoptosis through the PTEN(BIM)/Akt/Beclin1 pathway.

\section{miR-17-5p preserved kidney function}

After the administration of miR-17-5p agomir, the expression of miR-17-5p in kidney tissues significantly increased (see Figure 5A). Further, the miR-17-5p agomir significantly improved kidney function in the treatment group $24 \mathrm{~h}$ and $48 \mathrm{~h}$ after modeling, as indicated by the reduced Scr and BUN levels (see Figure 5B). The tissue analysis (see Figure 5C) also showed ameliorated tissue injury and reduced apoptosis in the miR-17-5p agomir treated group. The miR-17-5p agomir significantly reduced the autophagy level (see Figure 5D) and autophagosomes in the miR-17-5p treatment group (see Figure 5E). Further, the miR-17-5p agomir downregulated the overexpression of PTEN and BIM induced by kidney I/R (see Figure 5F). Additionally, miR-17-5p agomir treatment did not change the expression of Akt and Beclin1, but did upregulate the expression of p-Akt, p-Beclin1 (234), and p-Beclin1 (295) (see Figure 5G). The 7-day follow-up showed that I/R injury resulted in increased Scr and BUN and peaked on day 3. Notably, Scr and BUN levels in the I/R group were 3 and 4 times higher than those in the sham-operated group at day 7, respectively. Conversely, the Scr and BUN levels in the miR-17-5p group began to decrease on day 1 and almost completely recovered by day 7 (see Figure 5H).

\section{Discussion}

This study showed that the expression of $\mathrm{miR}-17-5 \mathrm{p}$ in kidney cortical tissues was significantly increased in the I/R group. miR-17-5p has been reported to increase in response to ischemia and hypoxia in various conditions. Research has shown that the expression of miR-17-5p is significantly upregulated in hypoxia-treated vascular smooth muscle cells (32), lung tissues from patients with pulmonary arterial hypertension (33), I/R injured hearts (34) and kidneys $(35,36)$. The overexpression of miR-17-5p by the miR$17-5 \mathrm{p}$ agomir improved kidney function and increased the survival of HK-2 cells, while the inhibition of miR-17-5p by the miR-17-5p antagomir exacerbated the injury. These results confirm the reno-protective effect of miR-17-5p. Additionally, we found that autophagy and apoptosis were significantly induced in the kidney I/R injury and cultured HK-2 cells that underwent hypoxia, and the overexpression of miR-17-5p appears to play a protective role by suppressing autophagy and apoptosis. Further, this study showed that miR-17-5p directly targeted PTEN and BIM, and the inhibition of PTEN and BIM further activated Akt/ Beclin1 signaling, which in turn reduced the autophagy level and attenuated kidney I/R injury.

The activation of autophagy plays an essential role in myocardial and liver I/R injury, and the inhibition of autophagy appears to protect myocardial cells and liver tissues from ischemic injury (37-39). I/R-induced hypoxic/ oxidative stress has been shown to enhance autophagy in different rodent kidney I/R models $(22,40,41)$. The pathological role of autophagy in hypoxia-induced kidney cell injury and kidney I/R injury was observed in this study. In the present study, the LC3II/LC3I ratio of the hypoxic HK-2 cells was increased by the prolongation of the reoxygenation time, while the viability and proliferation of the HK-2 cells were significantly impaired. However, the exact role of autophagy in kidney I/R remains unclear. In a bilateral kidney I/R mice model, blocking autophagy sensitized cultured tubular cells to hypoxia-induced apoptosis and resulted in severe kidney tissue injury (42). 

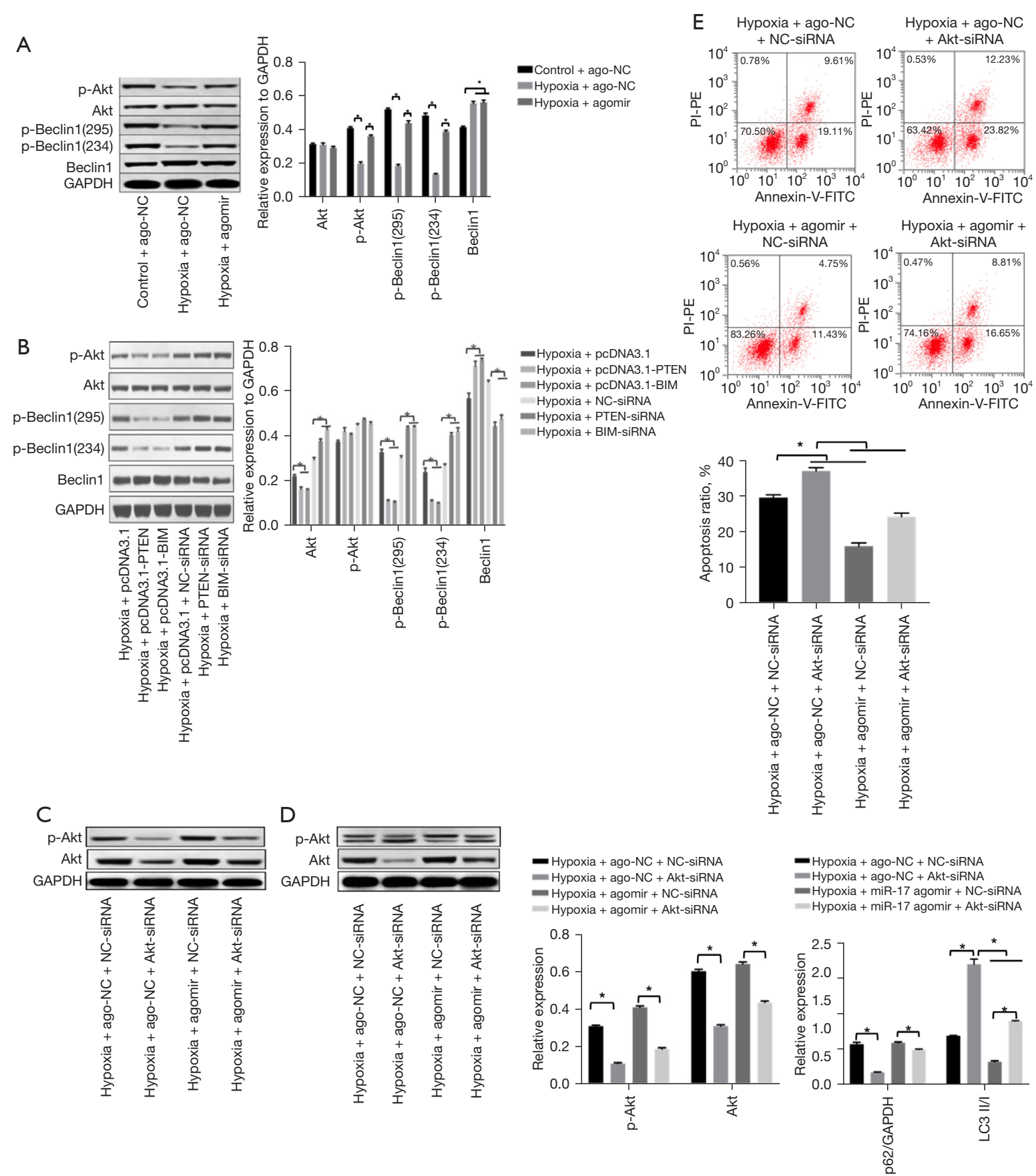

Figure 4 The mechanism of miR-17-5p is related to the Akt/Beclin1 signaling pathway. Representative band and statistical analysis (A) of p-Akt, Akt, Beclin1, p-Beclin1(234), and p-Beclin1 (295) expression among HK-2 cells treated with hypoxia or the miR-17-5p agomir. Representative band and statistical analysis (B) of p-Akt, Akt, Beclin1, p-Beclin1 (234), and p-Beclin1 (295) expression among HK-2 cells, which were treated with hypoxia, pcDNA3.1-PTEN, pcDNA3.1-BIM, or related siRNAs. Western blot (C) confirmed that the miR-17-5p agomir promoted the expression of p-AKT and Akt, while the Akt-siRNA reversed the upregulation. Akt-siRNA significantly increased the LC3II/I ratio and decreased the expression of p62, while the miR-17-5p agomir reversed this effect (D). Flow cytometry suggested that AktsiRNA greatly increased the apoptosis of $\mathrm{HK}-2$ cells. *, $\mathrm{P}<0.05$. ago-NC, agomir-negative control; Akt, protein kinase B; BIM, Bcl-2 like protein 11; GAPDH, glyceraldehyde phosphate dehydrogenase; HK-2, human kidney-2; LC3, microtubule-associated protein light chain 3; PTEN, phosphatase and tensin homolog deleted on chromosome 10; siRNA, small interfering RNA. 

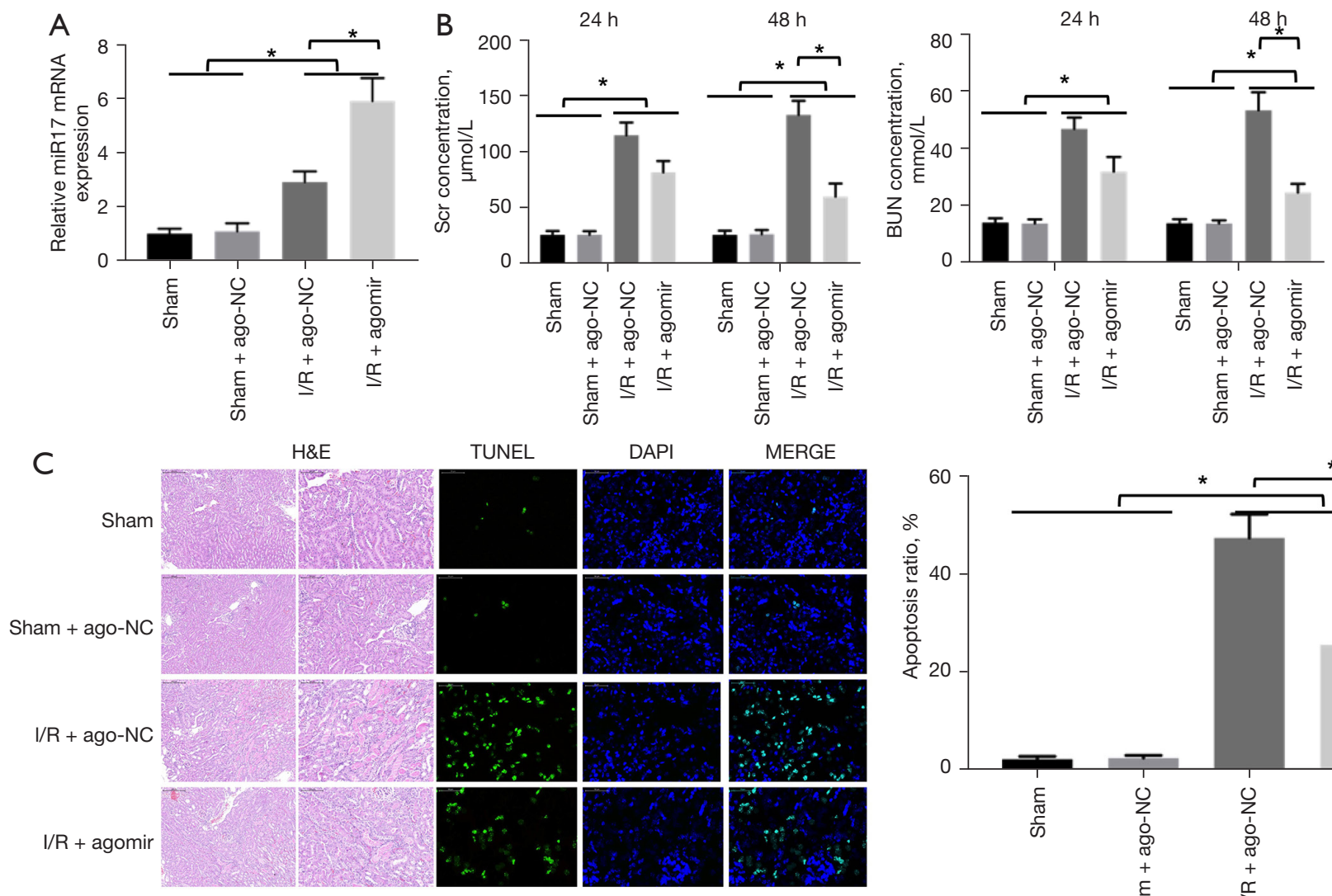

TUNEL
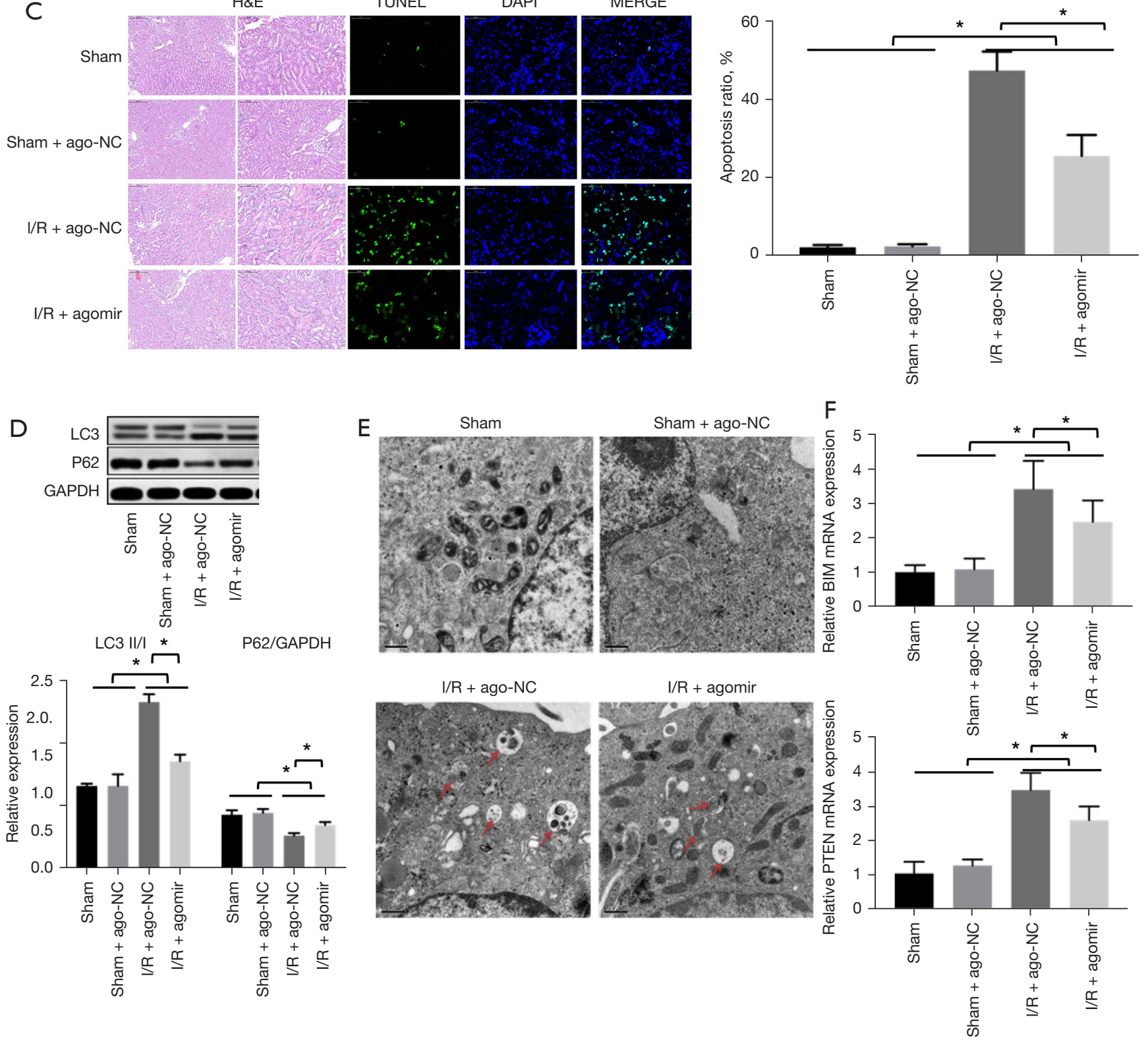

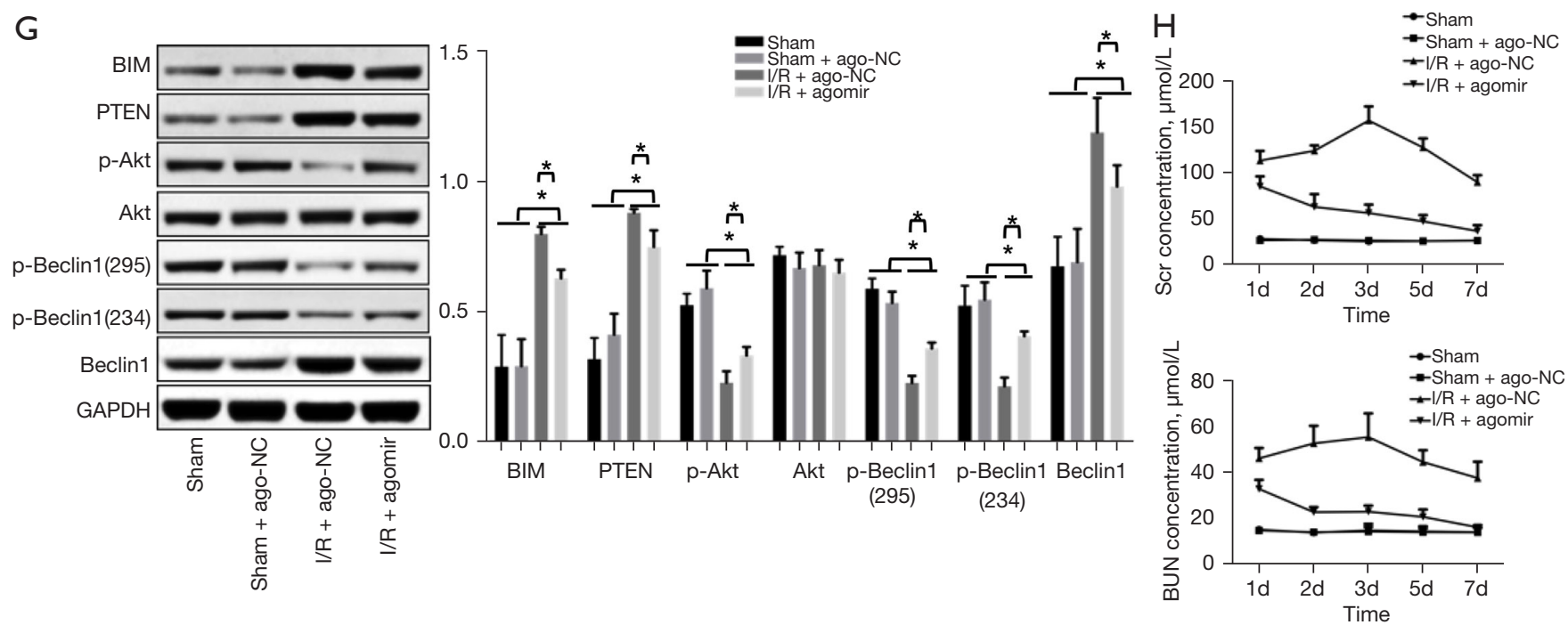

Figure 5 The miR-17-5p agomir was administrated via the tail vein $24 \mathrm{~h}$ before $\mathrm{I} / \mathrm{R}$ modeling ( $\mathrm{n}=4$ per group). The expression of miR-17$5 \mathrm{p}$ in mice kidneys (A), and the concentration of BUN and Scr (B) in mice after $24 \mathrm{~h}$ and $48 \mathrm{~h}$ of intervention. Representative images of kidney histology (C) with H\&E and TUNEL staining, and the statistical analysis of apoptosis in kidneys. Representative band and statistical analysis (D) of LC3B II/I and P62 expression in mice kidneys. Representative images of autophagosome and autophagolysosome (E) in mice kidneys detected by TEM, and the red arrow stands for autophagosome and autophagolysosome. The mRNA expression of BIM and PTEN in mice kidneys were detected by real-time PCR (F). Representative band and statistical analysis (G) of BIM, PTEN, p-Akt, Akt, Beclin1, p-Beclin1 (234), and p-Beclin1 (295) expression in mice kidneys. Trends in the concentrations of BUN and Scr $(\mathrm{H})$ in mice for 7 days postoperative follow-up. ${ }^{*}, \mathrm{P}<0.05$. ago-NC, agomir-negative control; Akt, protein kinase B; BIM, Bcl-2 like protein 11; BUN, blood urea nitrogen; DAPI, 4',6-diamidino-2-phenylindole; GAPDH, glyceraldehyde phosphate dehydrogenase; H\&E, hematoxylin-eosin; I/R, ischemia and reperfusion; LC3, microtubule-associated protein light chain 3; PTEN, phosphatase and tensin homolog deleted on chromosome 10; Scr, serum creatinine; TEM, transmission electron microscopy; TUNEL, terminal deoxynucleotidyl transferase-mediated dUTP nick-end labeling.

Additional effect of autophagy on kidney I/R injury was obtained from a bilateral kidney ischemia model using conditional proximal tubule Atg7 knockout mice (43). In a bilateral kidney ischemia rat model, the mTOR inhibitor everolimus was shown to stimulate autophagy and aggravate kidney I/R injury after 40 mins of ischemia (44). In 2 rat models of unilateral kidney ischemia, repetitive hypoxic preconditioning was shown to preserve kidney function and suppress I/R-induced tubular apoptosis and autophagy $(45,46)$. It has been proposed that autophagy in kidney I/ $\mathrm{R}$ injury has a dual role (protective and detrimental) that is largely dependent on the severity of the injury (47). In the present study, the autophagy inhibitor CQ significantly reduced the hypoxia-induced autophagy and apoptosis in the HK-2 cells, and the miR-17-5p-agomir mimicked the exact effect of CQ. A synergistic effect was exerted when miR-17-5p and CQ were combined, which might be due to the autophagy inhibition mechanism rather than lysosomes blocking the fusion of autophagosomes.

miR-17-5p has been reported to target the BMPR2, PTEN, and BIM pathways, which are involved in regulating autophagy (22-28). This study found a significant decrease in BIM and PTEN expression in the miR-17$5 \mathrm{p}$ agomir-treated HK-2 cells. PTEN can play a role in inhibiting Akt activation and blocking Akt-regulated downstream signaling events by dephosphorylating and converting phosphatidylinositol-3,4,5-trisphosphate to phosphatidylinositol-4,5-bisphosphate, which in turn promotes cell autophagy and apoptosis (48). The bioinformatic analysis indicated that miRNAs play crucial roles by directly binding to targeting the 3'UTRs of mRNAs and inhibiting gene expression. The luciferase reporter assay confirmed that PTEN is a direct target of $\mathrm{miR}-17-5 \mathrm{p}$, and is negatively regulated by miR-17-5p. Targeting PTEN suppresses the autophagy level in diabetic nephropathy, and the downstream PI3K/Akt pathway 
mediates this process $(48,49)$. In this study, silencing PTEN with a siRNA significantly attenuated the effect of miR-17$5 p$ inhibition on the hypoxia-induced apoptosis of HK-2 cells. Expression of phosphorylated Akt, as a downstream molecule of PTEN, is upregulated upon overexpression of miR-17-5p or silencing of PTEN with siRNA. On the other hand, silencing Akt with a siRNA significantly aggravated the hypoxia-induced apoptosis and autophagy of HK-2 cells. Furthermore, as a generally recognized antiautophagy protein, BIM recruits Beclin1 to microtubules by bridging Beclin1 and DYNLL1 to directly inhibit Beclin1 action; however, this interaction is disrupted by BIM phosphorylation in response to stress, which attenuates the inhibition of autophagy $(50,51)$. In a model of Parkinson's disease, the upregulated expression of miR-124 reduced autophagy by inhibiting BIM, probably due to the alleviation of the BIM-mediated Bax translocation to both lysosomal and mitochondrial membranes (52). In this study, overexpression of miR-17-5p downregulated the expression of BIM and attenuated kidney I/R injury by reducing the autophagy level, while the protective effect was reversed by silencing BIM with a siRNA. Beclin1 is the pivotal molecule in autophagy and has several phosphorylation sites with different autophagy effects (53). Countering the general conception that the phosphorylation of Beclin1 dissociates its interaction to Bcl-2 and induces autophagy, we found that Akt mediated phosphorylation at S234 and S295 leads to the suppression of Beclin1 function in autophagy and oncogenesis. A novel signaling pathway is proposed that inhibits PTEN and BIM by overexpressing miR-17-5p, which in turn upregulates Akt/Beclin1 and consequently inhibits I/R-induced autophagy and apoptosis.

This study had some limitations. First, there are significant anatomical and pathophysiological differences between mice and humans; thus, the results of this study need to be verified by more experiments. Second, we did not explore the effect of miR-17-5p on vascular endothelial cells, pericytes, or mesenchymal cells; thus, further in-depth subsequent need to be conducted.

\section{Conclusions}

miR-17-5p was identified as a novel protective gene to attenuate kidney ischemia and I/R injury. The expression of miR-17-5p is upregulated in kidney ischemia and I/ R. The upregulation of miR-17-5p expression inhibits apoptosis and autophagy by suppressing PTEN and BIM expression, which in turn upregulates downstream Akt/
Beclin1 expression. These findings suggest a new possible therapeutic strategy for the prevention and treatment of kidney I/R injury.

\section{Acknowledgments}

Funding: The work was supported by the Natural Science Foundation of China (Nos. 81870513, 81470980, and 81600584), the 1.3.5 Project for Disciplines of Excellence, West China Hospital, Sichuan University (ZY2016104), Youth Researcher Funding of Sichuan University (No. 2017SCU11042), and Research Funding of Sichuan Health and Family Planning Commission (Nos. 17PJ159, 18PJ434, and 18PJ453).

\section{Footnote}

Reporting Checklist: The authors have completed the ARRIVE reporting checklist. Available at https://dx.doi. org/10.21037/atm-21-4678

Data Sharing Statement: Available at https://dx.doi. org/10.21037/atm-21-4678

Conflicts of Interest: All authors have completed the ICMJE uniform disclosure form (available at https://dx.doi. org/10.21037/atm-21-4678). The authors have no conflicts of interest to declare.

Ethical Statement: The authors are accountable for all aspects of the work in ensuring that questions related to the accuracy or integrity of any part of the work are appropriately investigated and resolved. The Animal Ethics Committee of West China Hospital, Sichuan University, Chengdu, China (No. 2019141A) approved this study. All animal studies were conducted in accordance with the Guide for the Care and Use of Laboratory Animals of the National Institutes of Health.

Open Access Statement: This is an Open Access article distributed in accordance with the Creative Commons Attribution-NonCommercial-NoDerivs 4.0 International License (CC BY-NC-ND 4.0), which permits the noncommercial replication and distribution of the article with the strict proviso that no changes or edits are made and the original work is properly cited (including links to both the formal publication through the relevant DOI and the license). See: https://creativecommons.org/licenses/by-nc-nd/4.0/. 


\section{References}

1. Klaus F, Keitel da Silva C, Meinerz G, et al. Acute kidney injury after liver transplantation: incidence and mortality. Transplant Proc 2014;46:1819-21.

2. Engoren M, Habib RH, Arslanian-Engoren C, et al. The effect of acute kidney injury and discharge creatinine level on mortality following cardiac surgery*. Crit Care Med 2014;42:2069-74.

3. Venkatachalam MA, Weinberg JM, Kriz W, et al. Failed Tubule Recovery, AKI-CKD Transition, and Kidney Disease Progression. J Am Soc Nephrol 2015;26:1765-76.

4. Barba J, Zudaire JJ, Robles JE, et al. Is there a safe cold ischemia time interval for the renal graft?. Actas Urol Esp 2011;35:475-80.

5. Situmorang GR, Sheerin NS. Ischaemia reperfusion injury: mechanisms of progression to chronic graft dysfunction. Pediatr Nephrol 2019;34:951-63.

6. Tang C, Livingston MJ, Liu Z, et al. Autophagy in kidney homeostasis and disease. Nat Rev Nephrol 2020;16:489-508.

7. Chen W, Ruan Y, Zhao S, et al. MicroRNA-205 inhibits the apoptosis of renal tubular epithelial cells via the PTEN/Akt pathway in renal ischemia-reperfusion injury. Am J Transl Res 2019;11:7364-75.

8. Tang C, Han H, Yan M, et al. PINK1-PRKN/PARK2 pathway of mitophagy is activated to protect against renal ischemia-reperfusion injury. Autophagy 2018;14:880-97.

9. Chien CT, Shyue SK, Lai MK. Bcl-xL augmentation potentially reduces ischemia/reperfusion induced proximal and distal tubular apoptosis and autophagy. Transplantation 2007;84:1183-90.

10. Lempiäinen J, Finckenberg P, Mervaala EE, et al. Caloric restriction ameliorates kidney ischaemia/reperfusion injury through PGC-1 $\alpha$-eNOS pathway and enhanced autophagy. Acta Physiol (Oxf) 2013;208:410-21.

11. Decuypere JP, Ceulemans LJ, Agostinis P, et al. Autophagy and the Kidney: Implications for IschemiaReperfusion Injury and Therapy. Am J Kidney Dis 2015;66:699-709.

12. Nagalakshmi VK, Ren Q, Pugh MM, et al. Dicer regulates the development of nephrogenic and ureteric compartments in the mammalian kidney. Kidney Int 2011;79:317-30.

13. Sequeira-Lopez ML, Weatherford ET, Borges GR, et al. The microRNA-processing enzyme dicer maintains juxtaglomerular cells. J Am Soc Nephrol 2010;21:460-7.
14. Wang JF, Zha YF, Li HW, et al. Screening plasma miRNAs as biomarkers for renal ischemia-reperfusion injury in rats. Med Sci Monit 2014;20:283-9.

15. Wang IK, Sun KT, Tsai TH, et al. MiR-20a-5p mediates hypoxia-induced autophagy by targeting ATG16L1 in ischemic kidney injury. Life Sci 2015;136:133-41.

16. Bijkerk R, van Solingen C, de Boer HC, et al. Hematopoietic microRNA-126 protects against renal ischemia/reperfusion injury by promoting vascular integrity. J Am Soc Nephrol 2014;25:1710-22.

17. Wei Q, Liu Y, Liu P, et al. MicroRNA-489 Induction by Hypoxia-Inducible Factor-1 Protects against Ischemic Kidney Injury. J Am Soc Nephrol 2016;27:2784-96.

18. Marrone AK, Stolz DB, Bastacky SI, et al. MicroRNA-17 92 is required for nephrogenesis and renal function. J Am Soc Nephrol 2014;25:1440-52.

19. Patel V, Williams D, Hajarnis S, et al. miR-17 92 miRNA cluster promotes kidney cyst growth in polycystic kidney disease. Proc Natl Acad Sci U S A 2013;110:10765-70.

20. Hajarnis S, Lakhia R, Yheskel M, et al. microRNA-17 family promotes polycystic kidney disease progression through modulation of mitochondrial metabolism. Nat Commun 2017;8:14395.

21. Song T, Chen M, Rao Z, et al. miR-17-92 ameliorates renal ischemia reperfusion injury. Kaohsiung J Med Sci 2018;34:263-73.

22. Kimura T, Takabatake Y, Takahashi A, et al. Autophagy protects the proximal tubule from degeneration and acute ischemic injury. J Am Soc Nephrol 2011;22:902-13.

23. Long L, Yang X, Southwood M, et al. Chloroquine prevents progression of experimental pulmonary hypertension via inhibition of autophagy and lysosomal bone morphogenetic protein type II receptor degradation. Circ Res 2013;112:1159-70.

24. Chen S, Zhang Y, Zhou L, et al. A Bim-targeting strategy overcomes adaptive bortezomib resistance in myeloma through a novel link between autophagy and apoptosis. Blood 2014;124:2687-97.

25. Song BQ, Chi Y, Li X, et al. Inhibition of Notch Signaling Promotes the Adipogenic Differentiation of Mesenchymal Stem Cells Through Autophagy Activation and PTENPI3K/AKT/mTOR Pathway. Cell Physiol Biochem 2015;36:1991-2002.

26. $\mathrm{Hu} \mathrm{H}, \mathrm{Li} \mathrm{H}, \mathrm{He}$ Y. MicroRNA-17 downregulates expression of the PTEN gene to promote the occurrence and development of adenomyosis. Exp Ther Med 2017;14:3805-11.

27. Weng H, Huang H, Dong B, et al. Inhibition of miR-17 
and miR-20a by oridonin triggers apoptosis and reverses chemoresistance by derepressing BIM-S. Cancer Res 2014;74:4409-19.

28. Larabee SM, Coia H, Jones S, et al. miRNA-17 members that target Bmpr2 influence signaling mechanisms important for embryonic stem cell differentiation in vitro and gastrulation in embryos. Stem Cells Dev 2015;24:354-71.

29. Gong J, Wang X. Schisantherin A protects renal tubular epithelial cells from hypoxia/reoxygenation injury through the activation of PI3K/Akt signaling pathway. J Biochem Mol Toxicol 2018. [Epub ahead of print]. doi: 10.1002/ jbt.22160.

30. Hu S, Zhang Y, Zhang M, et al. Aloperine Protects Mice against Ischemia-Reperfusion (IR)-Induced Renal Injury by Regulating PI3K/AKT/mTOR Signaling and AP-1 Activity. Mol Med 2016;21:912-23.

31. Wang RC, Wei Y, An Z, et al. Akt-mediated regulation of autophagy and tumorigenesis through Beclin 1 phosphorylation. Science 2012;338:956-9.

32. Hao MX, Wang X, Jiao KL. MicroRNA-17-5p mediates hypoxia-induced autophagy and inhibits apoptosis by targeting signal transducer and activator of transcription 3 in vascular smooth muscle cells. Exp Ther Med 2017;13:935-41.

33. Lu Z, Li S, Zhao S, et al. Upregulated miR-17 Regulates Hypoxia-Mediated Human Pulmonary Artery Smooth Muscle Cell Proliferation and Apoptosis by Targeting Mitofusin 2. Med Sci Monit 2016;22:3301-8.

34. Du W, Pan Z, Chen X, et al. By targeting Stat3 microRNA-17-5p promotes cardiomyocyte apoptosis in response to ischemia followed by reperfusion. Cell Physiol Biochem 2014;34:955-65.

35. Kaucsár T, Révész C, Godó M, et al. Activation of the miR-17 family and miR-21 during murine kidney ischemia-reperfusion injury. Nucleic Acid Ther 2013;23:344-54.

36. Hao J, Wei Q, Mei S, et al. Induction of microRNA-175 p by $\mathrm{p} 53$ protects against renal ischemia-reperfusion injury by targeting death receptor 6 . Kidney Int 2017;91:106-18.

37. Li X, Hu X, Wang J, et al. Inhibition of autophagy via activation of $\mathrm{PI} 3 \mathrm{~K} / \mathrm{Akt} / \mathrm{mTOR}$ pathway contributes to the protection of hesperidin against myocardial ischemia/ reperfusion injury. Int J Mol Med 2018;42:1917-24.

38. Yu SY, Dong B, Fang ZF, et al. Knockdown of lncRNA AK139328 alleviates myocardial ischaemia/reperfusion injury in diabetic mice via modulating miR-204-3p and inhibiting autophagy. J Cell Mol Med 2018;22:4886-98.

39. Cui Z, Li S, Liu Z, et al. Interferon Regulatory Factor 1 Activates Autophagy to Aggravate Hepatic IschemiaReperfusion Injury by Increasing High Mobility Group Box 1 Release. Cell Physiol Biochem 2018;48:328-38.

40. Liu S, Hartleben B, Kretz O, et al. Autophagy plays a critical role in kidney tubule maintenance, aging and ischemia-reperfusion injury. Autophagy 2012;8:826-37.

41. Suzuki C, Isaka Y, Takabatake Y, et al. Participation of autophagy in renal ischemia/reperfusion injury. Biochem Biophys Res Commun 2008;368:100-6.

42. Jiang M, Liu K, Luo J, et al. Autophagy is a renoprotective mechanism during in vitro hypoxia and in vivo ischemiareperfusion injury. Am J Pathol 2010;176:1181-92.

43. Jiang M, Wei Q, Dong G, et al. Autophagy in proximal tubules protects against acute kidney injury. Kidney Int 2012;82:1271-83.

44. Nakagawa S, Nishihara K, Inui K, et al. Involvement of autophagy in the pharmacological effects of the mTOR inhibitor everolimus in acute kidney injury. Eur J Pharmacol 2012;696:143-54.

45. Wu HH, Hsiao TY, Chien CT, et al. Ischemic conditioning by short periods of reperfusion attenuates renal ischemia/reperfusion induced apoptosis and autophagy in the rat. J Biomed Sci 2009;16:19.

46. Yeh CH, Hsu SP, Yang CC, et al. Hypoxic preconditioning reinforces HIF-alpha-dependent HSP70 signaling to reduce ischemic renal failure-induced renal tubular apoptosis and autophagy. Life Sci 2010;86:115-23.

47. Sciarretta S, Hariharan N, Monden Y, et al. Is autophagy in response to ischemia and reperfusion protective or detrimental for the heart? Pediatr Cardiol 2011;32:275-81.

48. Song MS, Salmena L, Pandolfi PP. The functions and regulation of the PTEN tumour suppressor. Nat Rev Mol Cell Biol 2012;13:283-96.

49. Zhang Y, Zhao S, Wu D, et al. MicroRNA-22 Promotes Renal Tubulointerstitial Fibrosis by Targeting PTEN and Suppressing Autophagy in Diabetic Nephropathy. J Diabetes Res 2018;2018:4728645.

50. Luo S, Rubinsztein DC. BCL2L11/BIM: a novel molecular link between autophagy and apoptosis. Autophagy 2013;9:104-5.

51. Wang H, Ye Y, Zhu Z, et al. MiR-124 Regulates Apoptosis and Autophagy Process in MPTP Model of Parkinson's Disease by Targeting to Bim. Brain Pathol 2016;26:167-76.

52. Bové J, Martínez-Vicente M, Dehay B, et al. BAX channel activity mediates lysosomal disruption linked to Parkinson 
Page 16 of 16

disease. Autophagy 2014;10:889-900.

53. Menon MB, Dhamija S. Beclin 1 Phosphorylation - at the Center of Autophagy Regulation. Front Cell Dev Biol

Cite this article as: $\mathrm{Ma} M, \mathrm{Fu} \mathrm{L}$, Jia Z, Zhong Q, Huang Z, Wang X, Fan Y, Lin T, Song T. miR-17-5p attenuates kidney ischemia-reperfusion injury by inhibiting the PTEN and BIM pathways. Ann Transl Med 2021;9(20):1545. doi: 10.21037/atm$21-4678$
Ma et al. The pathway of miR-17-5p attenuates kidney IRI

2018;6:137.

(English Language Editor: L. Huleatt) 
Supplementary

Table S1 Primer for quantitative real-time polymerase chain reaction (qRT-PCR)

\begin{tabular}{ll}
\hline Gene & Sequence \\
\hline miR-17-5p & F: ACACTCCAGCTGGGCAAAGTGCTTACAGTGC; R: CTCAACTGGTGTCGTGGAGTCGGCAATTCAGTTGAGCTACCTGC \\
U6 & F: CTCGCTTCGGCAGCACA; R: AACGCTTCACGAATTTGCGT \\
PTEN & F: TGGATTCGACTTAGACTTGACCT; R: TACGCCTTCAAGTCTTTCTGC \\
GAPDH & F: GAAGGTGAAGGTCGGAGTC; R: GAAGATGGTGATGGGATTTC \\
BMPR2 & F: GAATCCCCAGCCCTGAAACA; R: TACTTGGCGTGAGTCCTGTG \\
BIM & F: GCATCATCGCGGTATTCGGT; R: GAACAAGGGCCAAGAAA GGG
\end{tabular}

BIM, Bcl-2 like protein 11; BMPR2, bone morphogenetic protein type II receptor; GAPDH, glyceraldehyde phosphate dehydrogenase; PTEN, phosphatase and tensin homolog deleted on chromosome 10.

Table S2 Primer for Luciferase reporter assay

\begin{tabular}{ll} 
Molecules & Sequence of primer \\
\hline PTEN-3'UTR & F: TAGGCGATCGCTCGAGACCCTATACATCCACAGGG; R: TTGCGGCCAGCGGCCGCGTGAACCAGTATATCACAAAG \\
BIM-3'UTR & F: TAGGCGATCGCTCGAGCGGTTACAAGCAGTGGTAG; R: TTGCGGCCAGCGGCCGCGGAAATGTGCTCATGTGGTG
\end{tabular}

BIM, Bcl-2 like protein 11; PTEN, phosphatase and tensin homolog deleted on chromosome 10. 
Table S3 Sequence of related siRNAs

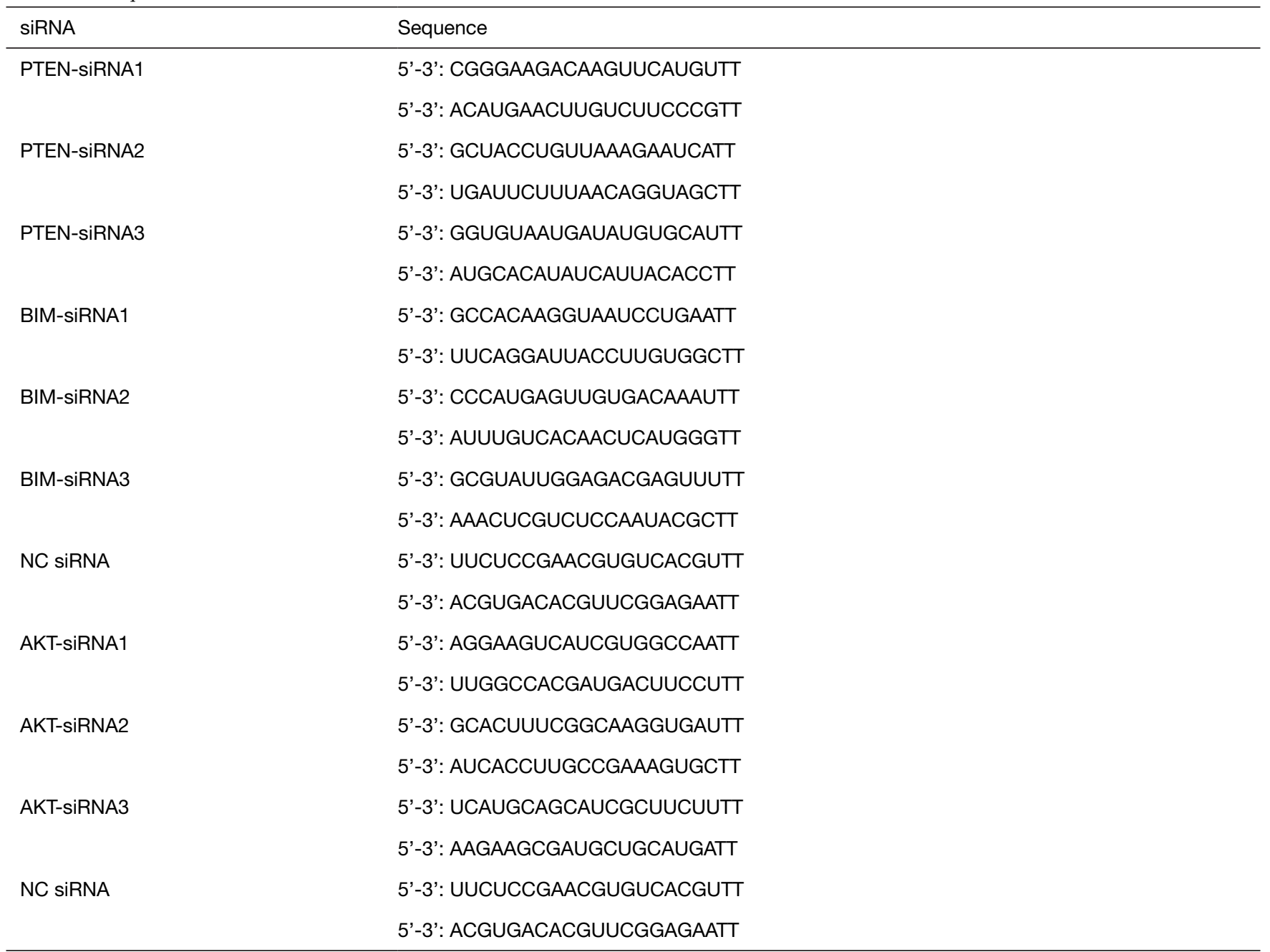

Akt, protein kinase B; BIM, Bcl-2 like protein 11; BMPR2, bone morphogenetic protein type II receptor; GAPDH, glyceraldehyde phosphate dehydrogenase; NC, negative control; PTEN, phosphatase and tensin homolog deleted on chromosome 10; siRNA, small interfering RNA.

Table S4 Sequence of agomir and antagomir

\begin{tabular}{ll}
\hline Agents & Sequence \\
\hline miR-17-5p agomir & 5'-3': CAAAGUGCUUACAGUGCAGGUAG \\
& 5'-3': ACCUGCACUGUAAGCACUUUGUU \\
miR-17-5p antagomir & 5'-3': CUACCUGCACUGUAAGCACUUUG \\
agomir NC & 5'-3': UUCUCCGAACGUGUCACGUTT \\
& 5'-3': ACGUGACACGUUCGGAGAATT \\
antagomir NC & 5'-3': CAGUACUUUUGUGUAGUACAA \\
\hline
\end{tabular}

NC, negative control. 